\title{
Primary solitary fibrous tumor of the thyroid gland: A review starting from a case report
}

\author{
Ion Negură ${ }^{1,2, *}$, Victor lanole ${ }^{2}$, Radu Dănilă${ }^{3}$, Maria-Christina Ungureanu ${ }^{4}$, Delia \\ Gabriela Ciobanu Apostol ${ }^{1,2}$
}

${ }^{1}$ Pathology Department, "Sf. Spiridon" Emergency County Clinical Hospital, lasi, Romania; ${ }^{2}$ Pathology Department, "Grigore T. Popa" University of Medicine and Pharmacy, lasi, Romania; ${ }^{3}$ Department of Surgery, III-rd Surgical Unit, "Sf. Spiridon" Emergency County Clinical Hospital, "Grigore T. Popa" University of Medicine and Pharmacy, lasi, Romania; "Endocrinology Department, "Sf. Spiridon" Emergency County Clinical Hospital, "Grigore T. Popa" University of Medicine and Pharmacy, lasi, Romania

\begin{abstract}
Primary solitary fibrous tumor (SFT) of the thyroid gland is a rare mesenchymal tumor with fibroblastic differentiation, ramified, thin-walled, enlarged (staghorn) vessels and specific NAB2-STAT6 gene fusion, which is more commonly found in pleura and peritoneum. This neoplasm can be located in a variety of anatomical sites outside pleura and peritoneum including bone, visceral organs and soft tissues, head and neck examples representing only $10-15 \%$ of the extra-pleural and extra-peritoneal tumors. Diagnosing this entity can be difficult, especially in thyroid gland, mainly because of the rarity of this neoplasm, but presence of characteristic microscopic features together with positivity for STAT6 and CD34 can confirm the diagnosis and exclude other differential diagnosis. Information about the diagnosis and treatment options of thyroid SFTs is limited but almost all primary thyroid SFTs have a good prognosis and indolent clinical course. Clinical surveillance is still necessary because some SFTs can be aggressive. Raising awareness regarding extra-pleural and extraperitoneal location of this tumor in endocrine organs can help to better manage these patients. We report the case of a 34-year-old female with primary SFT of the thyroid gland. Additionally, we review the literature for the main clinical, paraclinical and pathological features of this neoplasm.
\end{abstract}

Keywords: solitary fibrous tumor; thyroid; mesenchymal neoplasm; CD34; BCL-2; STAT6

\section{Introduction}

Solitary fibrous tumor (SFT) represents a rare mesenchymal soft tissue neoplasm with fibroblastic differentiation [1], specific NAB2STAT6 gene fusion [2] and characteristic ramified, thin-walled, enlarged (staghorn) vascularization [3], which was first described as a pleural tumor in 1931 by Klemperer and Rabin [4]. NAB2-STAT6 gene fusion correlates

Received: November 2021; Accepted after review: December 2021; Published: December 2021.

${ }^{*}$ Corresponding author: Ion Negură, Pathology Department, "Grigore T. Popa" University of Medicine and Pharmacy, lasi

Email: dr.neguraionut@gmail.com with nuclear expression of STAT6 immunomarker [5], which is the most sensitive and specific marker for positive diagnosis of SFT [6]. This neoplasm can be located in a variety of anatomical sites outside pleura and peritoneum including bone, visceral organs and soft tissues, head and neck examples representing less than $0.1 \%$ of all SFT [7]. Primary SFT of the thyroid gland is a rare tumor encountered in middle-aged patients with equal sex distribution [8,9]. The first three cases of primary SFT of the thyroid were reported in 1993 [10] and since then, to the best of our knowledge, only 39 cases have been published in English literature [11]. SFT usually presents in patients with euthyroidism [12] as a solid slow-growing cervical mass [13]. Microscopically, SFT has patternless 
growth pattern, variable cellularity, ectatic ramified vessels [14] and characteristic immunophenotype with expression of STAT6, CD34, CD99, BCL-2 and vimentin [15-19]. Diagnosing this neoplasm can prove a real challenge when STAT6 is not available, especially in extra-pleural and extra-peritoneal cases but the characteristic morphology together with positivity for other markers frequently expressed in SFT is enough for a positive diagnosis. Here we report a case of primary SFT of the thyroid gland, diagnosed in a 34-year-old female patient with a history of colloid goiter.

\section{Case presentation}

A 34-year-old female patient was initially referred to the Endocrinology Department with the diagnosis of multinodular goiter. Clinical and para-clinical examination revealed a solid hypoechogenic nodule measuring 3.5/2.9/2.3 $\mathrm{cm}$ in the left thyroid lobe associated with euthyroidism and normal biological tests. Fine needle aspiration biopsy (FNAB) under ultrasound guidance was performed, with a benign result, according to Bethesda classification.

Although no compression symptoms were present, due to the volume of the nodule surgical treatment was an option but the patient decided to postpone surgery and was discharged. After a four-year period of close follow-up, the same euthyroid status was present but an increase of nodule dimensions to $5.5 / 4.9 / 3 \mathrm{~cm}$, associated with compression signs and symptoms (wheezing, dyspnea and hoarseness) was noted and the patient was again referred to the surgical department. Although thyroid lobectomy was also considered, given the option of the patient a total thyroidectomy was performed. Intraoperative exploration revealed a solid nodule in the middle part of the left lobe and no enlarged lymph nodes in the central compartment. The postoperative course was uneventful and the patient was discharged after 2 days.

In the Pathology Department, we received the surgical specimen fixed in $10 \%$ neutral buffered formalin. Gross specimen examination revealed a well-circumscribed mass measuring $5.1 / 4.3 / 3 \mathrm{~cm}$ which involved left thyroid lobe. On cut section, a firm solid appearance with cystic spaces was noted. Microscopic examination identified a mesenchymal spindle cell proliferation with fascicular and storiform growth pattern with alternation of hypocellular and hypercellular areas (Figure 1A).

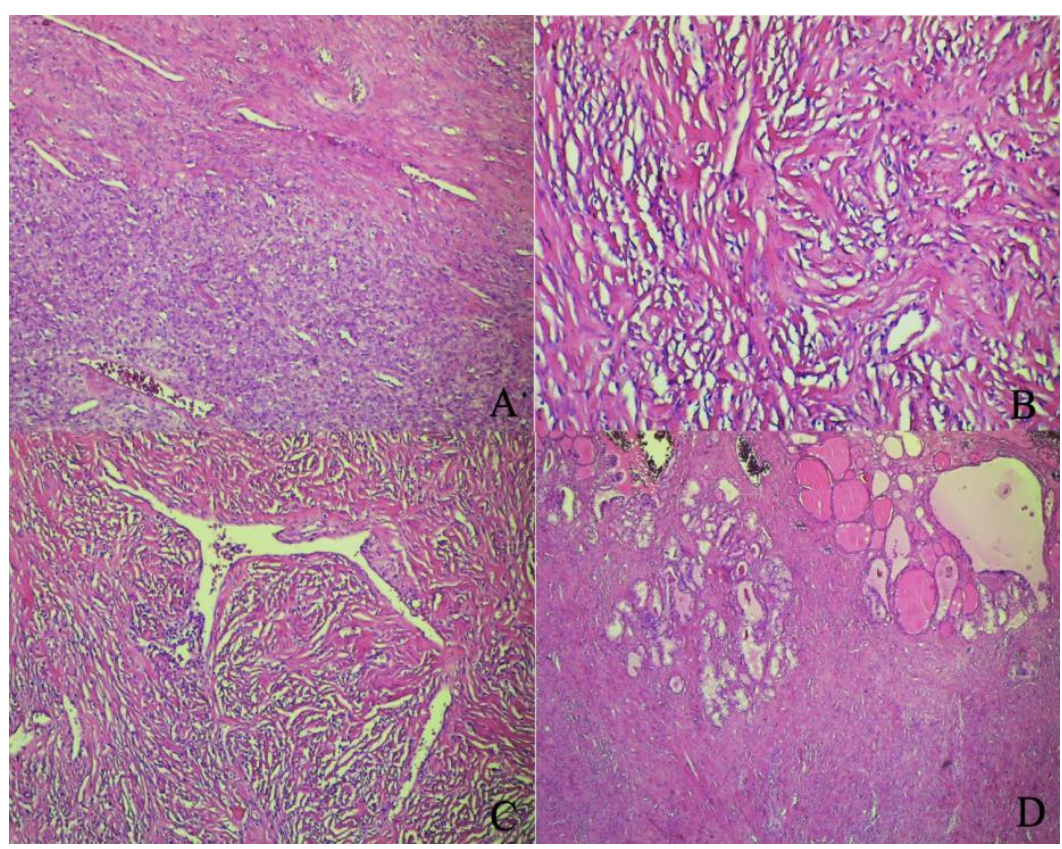

Fig. 1. Histomorphological aspects of thyroid SFT. A: Hypocellular and hypercellular areas (HE, x100); B: Abundant collagen deposits (HE, x200); C: Staghorn blood vessels (HE, x100); D: Entrapment of thyroid follicles (HE, x40). 
In hypocellular areas, abundant collagen deposits (Figure 1B) and prominent staghorn blood vessels were identified (Figure 1C). Tumor cells had scant eosinophilic cytoplasm, indistinct borders and bland nuclei. Mitotic activity and necrosis were absent. At the margins of the tumor, focal entrapment of thyroid follicles was present (Figure 1D). The residual gland had features of multinodular colloid goiter.

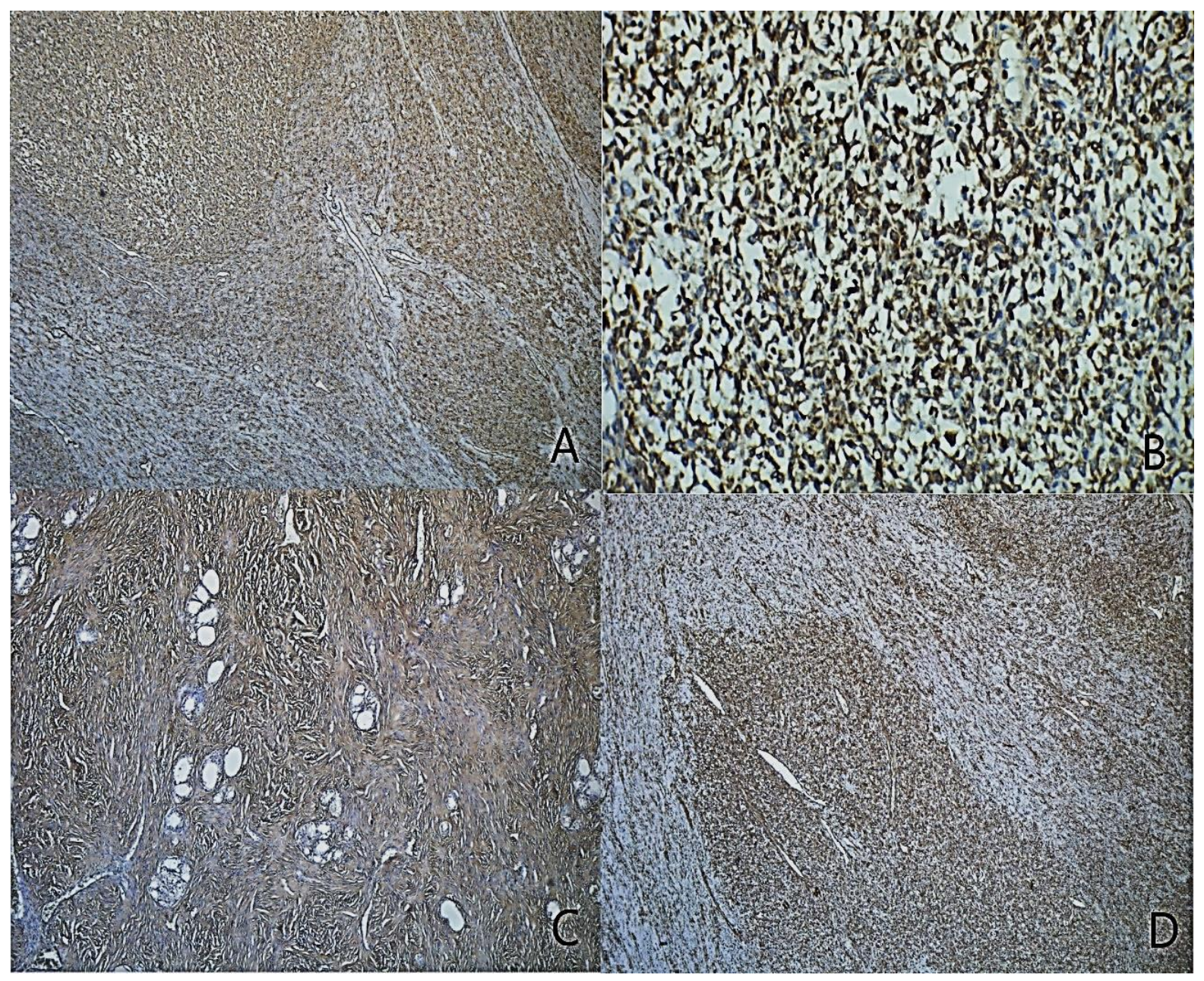

Fig. 2. Immunohistochemical profile of thyroid SFT. Strong and diffuse positivity of tumor cells for: A. CD34 (IHC, anti-CD34 antibody, x40); B. BCL-2 (IHC, anti-BCL-2 antibody, x200); C. CD99 (IHC, anti-CD99 antibody, x40);

D. vimentin (IHC, anti-vimentin antibody, x40).

On immunohistochemistry, neoplastic cells were CD34 (Figure 2A), BCL-2 (Figure 2B), CD99 (Figure 2C) and vimentin positive (Figure 2D) and negative for TTF-1 (Figure 3A), S100 (Figure 3B), CD68, NSE, desmin (Figure 3C) and SMA (Figure 3D) with a Ki-67 proliferation index $<1 \%$. Based on morphological and immunohistochemical features, the final diagnosis was primary solitary fibrous tumor of thyroid gland. 


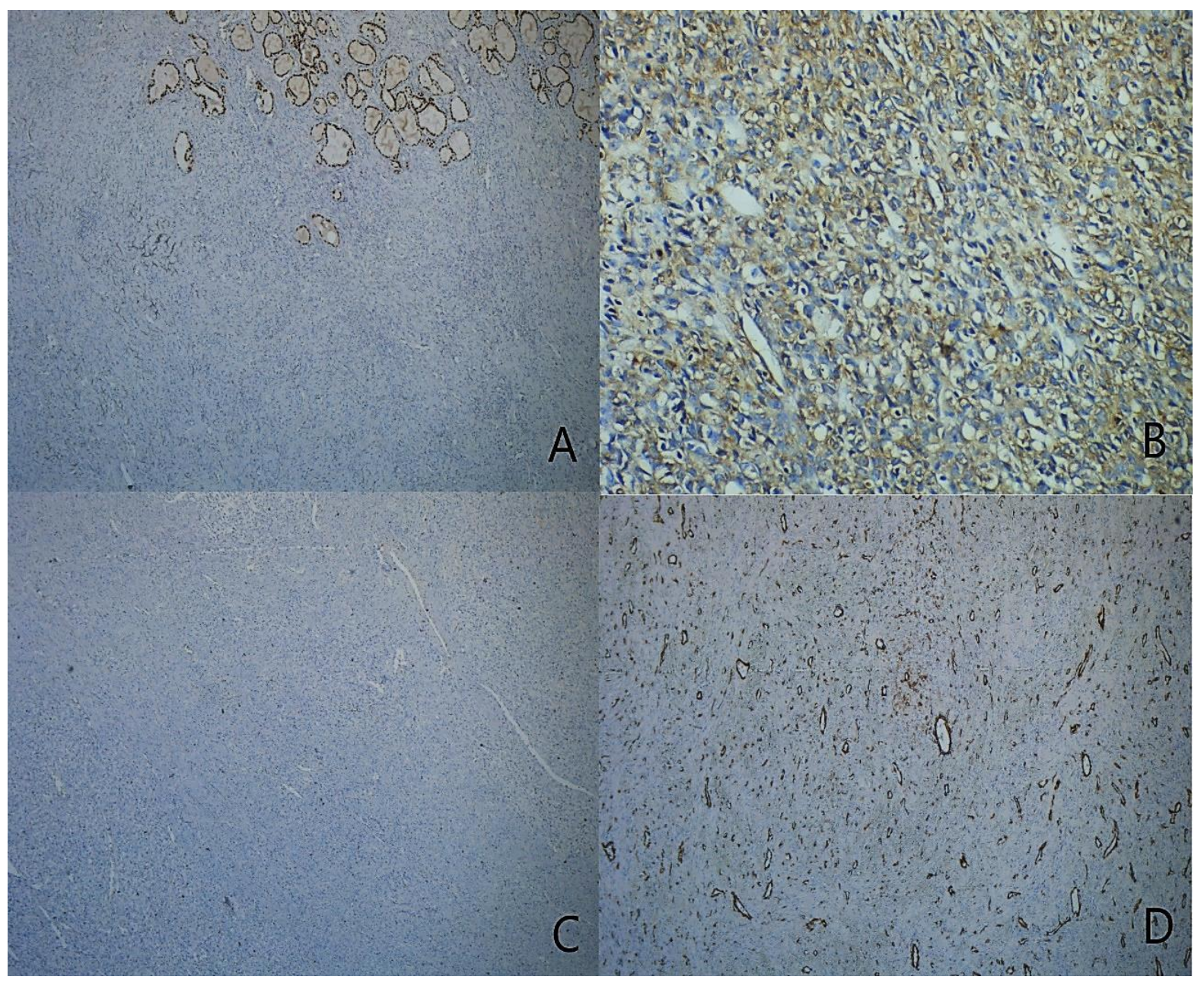

Fig. 3. Immunohistochemical profile of thyroid SFT. Total negativity for: A. TTF-1 in tumor cells, with positivity in thyroid follicular cells (IHC, anti-TTF-1 antibody, x40); B. S100 protein with aberrant cytoplasm positivity (IHC, anti-S100 antibody, x200); C. desmin (IHC, anti-desmin antibody, x40); D. SMA in tumor cells, with positivity in smooth muscle layer of blood vessels (IHC, anti-SMA antibody, x100).

\section{Discussion}

SFTs are rare mesenchymal tumors encountered mainly in pleura [20], but extrathoracic examples have been reported [21], the head and neck location, as in our case, being extremely rare [22]. The pathogeny of this tumor is not associated with any risk factors. Usually, primary thyroid SFT develops in middle-aged patients with no sex predilection [8] and the age at diagnosis varies between 28-88 years [9]. A slow-growing thyroid mass is usually the only clinical manifestation in primary thyroid SFT and is frequently associated with euthyroidism [13]. Similar to our case, thyroidectomy is performed in those rare situations with compressive symptoms or respiratory failure [7].

Ultrasound examination is the first investigation in case of thyroid masses [23], and when a SFT involves the thyroid gland, it identifies a solid, well-circumscribed nodule [12]. SFT presents on contrast enhanced computed tomography (CT) as a wellcircumscribed, lobulated mass that has a density similar to the skeletal muscle and heterogeneous contrast augmentation [1]. On magnetic resonance imaging (MRI) examination, fibrous areas have intermediate intensity on T1-weighted images while cellular or myxoid zones present as variable hypointensity to hyperintensity on T2-weighted images [1]. Necrosis, hemorrhage, cystic and myxoid changes or calcifications are frequently 
encountered in aggressive or large tumors and manifest as increased heterogeneity on MRI and CT examination [1]. In our case, ultrasound examination was the only imagistic examination used and initially identified a solid hypoechogenic nodule measuring 3.5/2.9/2.3 $\mathrm{cm}$ which increased in size to $5.5 / 4.9 / 3 \mathrm{~cm}$ over a period of four years.

Diagnosis of SFT on FNAB is extremely difficult because aspirates have low cellularity and are often nondiagnostic [24]. When FNAB contains spindle cells without atypia and small stromal collagen fragments, a diagnosis of SFT can be suspected [25]. Only when immunocytochemistry is performed and STAT6 is positive, a diagnosis of SFT can be made on FNAB [26]. In our case, FNAB performed at the initial presentation contained colloid, red blood cells, foamy macrophages, siderophages and benign thyroid follicular cells, but no spindle cells or stromal collagen fragments. Therefore, the smear was classified as benign according to Bethesda classification.

Macroscopically, primary thyroid SFT is a well-circumscribed nodule with a fibrous white or gray cut surface, sometimes associated with cystic areas [27]. Gross examination, in our report, identified a mass measuring $5.1 / 4.3 / 3 \mathrm{~cm}$, but tumor size may vary between $1.7-13.8 \mathrm{~cm}$, with a $5.7 \mathrm{~cm}$ median size [13]. SFTs of thyroid have similar histologic features with their counterparts involving pleura. The cellularity is variable, with hypo- and hypercellular areas composed of spindle cells without atypia and patternless growth arrangement. Stroma is collagenous with ramified dilated vessels. Thyroid follicles are often found entrapped in the tumor. This aspect does not represent true invasion and should be viewed as blending of the tumor with the follicles. Lipomatous, giant cell-rich and myxoid types represent histologic variants of SFT [16]. Only the lipomatous histologic variant of SFT was described in thyroid [28]. Nuclear STAT6 protein expression correlates with NAB2-STAT6 gene fusion which is pathognomonic for this neoplasm. The genetic hallmark alteration in SFT is a recurrent fusion on chromosome 12q13 spanning the loci of NAB2 and STAT6. STAT6 and NAB2 are transcriptional regulators and, in SFT, the early growth response (EGR)-binding domain of NAB2 is fused to the activating domain of STAT6 [29]. This NAB2-STAT6 fusion promotes nuclear relocation of STAT6 which can be immunohistochemically identified with anti-STAT6 antibody. Thus, strong and diffuse STAT6 nuclear expression is a reliable surrogate marker for NAB2-STAT6 gene fusion [29]. Other markers frequently expressed in SFT are CD34, BCL-2, CD99 and vimentin [17]. Primary thyroid SFT is negative for TTF-1, calcitonin, thyroglobulin, CD31, desmin, cytokeratins, CD117 and S100 protein [30]. In our case, the tumor was CD34, CD99, BCL-2 and vimentin positive and negative for TTF-1, S100, CD68, NSE, desmin and SMA with a Ki-67 proliferation index $<1 \%$, which correlates with typical SFT immunoprofile. STAT6 was not performed because it was not available in our laboratory, but morphological features and typical immunoprofile were sufficient to establish the diagnosis of primary SFT of the thyroid gland.

The differential diagnosis of thyroid SFT includes several other spindle cell tumors which can involve this organ. Epithelial neoplasms like papillary thyroid carcinoma, follicular adenoma and follicular carcinoma can sometimes have a spindled appearance, mimicking SFT. Spindle cells encountered in these tumors are considered to be metaplastic and are positive for TTF-1, cytokeratin and thyroglobulin and lack expression of STAT6 and CD34 [31]. A spindle cell component which can have overlapping features with SFT can be present in papillary carcinoma with desmoid-type fibromatosis, but this component is SMA positive and CD34 negative [32]. Monophasic variant of spindle epithelial tumor with thymus-like differentiation (SETTLE) has predominantly spindle cells areas and can be mistaken for SFT. The spindle cell component in this case is negative for STAT6 and CD34 [33]. Other mesenchymal tumors with spindle cell morphology are frequently confused with thyroid SFT. From this category, peripheral nerve sheath tumors and smooth muscle tumors should be excluded in order to establish a positive diagnosis of SFT [34, 35]. Leiomyosarcomas are the most frequent primary thyroid smooth muscle tumors [36]. SMA and desmin expression combined with 
absence of CD34 and STAT6 expression can differentiate smooth muscle tumors from SFT [37]. Before a diagnosis of SFT can be made, peripheral nerve sheath tumors must be ruled out. Neurofibromas, schwannomas and also malignant peripheral nerve sheath tumors have been reported in thyroid [38]. From this category of tumors, schwannomas are most often confused with SFTs because these tumors have similar morphology, with hyperand hypocellular areas (Antoni A and B zones), hyalinized stroma and ramified ectatic vessels [39]. Schwannomas are SOX10 [40] and S100 positive [41] whereas CD34 and STAT6 are not expressed by this category of peripheral nerve sheath tumors. Immunomarkers used in our case, in the differential diagnosis workup, successfully excluded epithelial neoplasms, SETTLE, peripheral nerve sheath tumors and smooth muscle tumors of the thyroid gland.

Surgical removal with negative margins is the best treatment for primary thyroid SFT [42]. Usually, there is no need for adjuvant therapies in SFT of the thyroid gland because, generally, SFTs are biologically indolent. SFT can sometimes recur [43], especially in those cases with positive surgical margins and only rarely can generate distant metastases [44]. Complete surgical resection with negative margins was performed in our patient case.

Hypercellularity, cytologic atypia, increased mitoses ( $>4$ mitoses per $2 \mathrm{~mm} 2$ ), tumor necrosis and infiltrative margins are considered to be histomorphological aspects of malignancy [45] and were absent in our patient case. However, SFT has a clinical evolution that is quite difficult to assess based only on histomorphological aspects. In order to predict clinical evolution in cases of SFT, risk stratification schemes that include variables such as tumor site, patient age, cellularity, pleomorphism, mitotic count and necrosis have been developed [46], but because primary thyroid SFT is a very rare tumor, the number of cases included in these schemes

\section{References}

1. Davanzo B, Emerson RE, Lisy M, Koniaris LG, Kays JK. Solitary fibrous tumor. Transl Gastroenterol Hepatol. 2018; 3:94. https://doi:org/10.21037/tgh.2018.11.02. was very low. In general, primary thyroid SFTs are associated with a favorable clinical evolution, but data regarding the evolution of SFT in other locations show that recurrences and metastases can develop after a five-year period [46]. Taking this into consideration, long-term close surveillance is advisable for these patients. Our patient is currently under clinical and paraclinical surveillance. Last medical check-up was in September 2020 and a euthyroid status under hormone replacement therapy with no recurrence was noted.

\section{Conclusions}

Primary thyroid SFT is a rare mesenchymal spindle cell neoplasm which is frequently encountered in middle-aged patients. Patients usually present a slowgrowing cervical mass that is mobile with deglutition and in rare situations, compressive symptoms can develop. Histological diagnosis of SFT can be difficult because thyroid spindle cell tumors are rarely encountered in day-today practice and because differential diagnosis includes numerous other spindle cell lesions. Immunohistochemistry is the only ancillary test that can differentiate SFT from other mimicking spindle cell lesions. A definitive diagnosis of SFT can be made if the tumor is STAT6 nuclear positive, but histomorphology together with other markers frequently expressed in this neoplasm are sufficient for positive and differential diagnosis. Prognosis in thyroid SFT is favorable, but because cases with late recurrence and metastases were reported, long-term surveillance is required.

\section{Conflicts of interest}

There are no personal, financial, or other conflicts of interest to disclose.

\section{Consent}

Written informed consent was obtained from the patient for publication of this case report.

2. Chmielecki J, Crago AM, Rosenberg M, et al. Whole-exome sequencing identifies a recurrent NAB2-STAT6 fusion in solitary fibrous tumors. 
Nat Genet. 2013; 45(2):131-132. https://doi.org/10.1038/ng.2522 .

3. Huang SC, Huang HY. Solitary fibrous tumor: An evolving and unifying entity with unsettled issues. Histol Histopathol. 2019; 34(4):313-334. https://doi.org/10.14670/HH-18-064.

4. Klemperer $P$, Rabin CB. Primary neoplasms of the pleura. A report of five cases. Arch Pathol. $1931 ; 11: 385-412$.

5. Doyle LA, Vivero M, Fletcher CD, Mertens F, Hornick JL. Nuclear expression of STAT6 distinguishes solitary fibrous tumor from histologic mimics. Mod Pathol. 2014; 27(3):390-395.

https://doi.org/10.1038/modpathol.2013.164.

6. Schweizer L, Koelsche C, Sahm F, et al. Meningeal hemangiopericytoma and solitary fibrous tumors carry the NAB2-STAT6 fusion and can be diagnosed by nuclear expression of STAT6 protein. Acta Neuropathol. 2013; 125(5):651-658.

https://doi.org/10.1007/s00401-013-1117-6

7. Larsen SR, Godballe C, Krogdahl A. Solitary fibrous tumor arising in an intrathoracic goiter. Thyroid. 2010; 20(4):435-437. https://doi.org/10.1089/thy.2009.0237

8. Suh YJ, Park JH, Jeon JH, Bilegsaikhan SE. Extrapleural solitary fibrous tumor of the thyroid gland: A case report and review of literature. World J Clin Cases. 2020; 8(4):782-789. https://doi.org/10.12998/wjcc.v8.i4.782.

9. Mizuuchi $\mathrm{Y}$, Yamamoto $\mathrm{H}$, Nakamura $\mathrm{K}$, et al. Solitary fibrous tumor of the thyroid gland. Med Mol Morphol. 2014; 47(2):117-122. https://doi.org/10.1007/s00795-013-0056-6

10. Taccagni G, Sambade C, Nesland J, Terreni MR, Sobrinho-Simões M. Solitary fibrous tumour of the thyroid: clinicopathological, immunohistochemical and ultrastructural study of three cases. Virchows Arch A Pathol Anat Histopathol. 1993; 422(6):491-497. https://doi.org/10.1007/BF01606459

11. Mohamed A, Qureshi F, Eldin RS. Solitary fibrous thyroid tumor: A case report. $J$ Endocr Soc. 2021; 5(1 Suppl):A900-901. https://doi.org/10.1210/jendso/bvab048.1838.

12. Papi G, Corrado S, Uberti ED, Roti E. Solitary fibrous tumor of the thyroid gland. Thyroid. 2007; 17(2):119-126. https://doi.org/10.1089/thy.2005.0202

13. Thompson LDR, Wei C, Rooper LM, Lau SK. Thyroid gland solitary fibrous tumor: report of 3 cases and a comprehensive review of the literature. Head Neck Pathol. 2019; 13(4):597$605 . \quad$ https://doi.org/10.1007/s12105-01901012-6

14. Song Z, Yu C, Song X, Wei L, Liu A. Primary solitary fibrous tumor of the thyroid - report of a case and review of the literature. $J$ Cancer. 2011; 2:206-209.

https://doi.org/10.7150/jca.2.206

15. Saeed O, Zhang S, Cheng L, Lin J, Alruwaii F, Chen S. STAT6 expression in solitary fibrous tumor and histologic mimics: a single institution experience. Appl Immunohistochem Mol Morphol. 2020; 28(4):311-315. https://doi.org/10.1097/PAl.0000000000000745

16. Tariq MU, Din NU, Abdul-Ghafar J, Park YK. The many faces of solitary fibrous tumor; diversity of histological features, differential diagnosis and role of molecular studies and surrogate markers in avoiding misdiagnosis and predicting the behavior. Diagn Pathol. 2021; 16(1):32. https://doi.org/10.1186/s13000021-01095-2

17. Han $Y$, Zhang $Q, Y u \quad X$, et al. Immunohistochemical detection of STAT6, CD34, CD99 and BCL-2 for diagnosing solitary fibrous tumors/hemangiopericytomas. Int $\mathrm{J}$ Clin Exp Pathol. 2015; 8(10):13166-13175. PMID:26722515.

18. Hasegawa $T$, Matsuno $\mathrm{Y}$, Shimoda $\mathrm{T}$, Hirohashi S, Hirose T, Sano T. Frequent expression of bcl-2 protein in solitary fibrous tumors. Jpn J Clin Oncol. 1998; 28(2):86-91. https://doi.org/10.1093/jico/28.2.86

19. Geramizadeh B, Marzban M, Churg A. Role of immunohistochemistry in the diagnosis of solitary fibrous tumor, a review. Iran $J$ Pathol. 2016; 11(3):195-203. PMID:27799967.

20. Mordenti $P$, Di Cicilia R, Delfanti $R$, Capelli $P$, Paties C, Cavanna L. Solitary fibrous tumors of the pleura: a case report and review of the literature. Tumori. 2013; 99(4):e177-183. https://doi.org/10.1700/1361.15120

21. Ronchi A, Cozzolino I, Zito Marino F, et al. Extrapleural solitary fibrous tumor: A distinct entity from pleural solitary fibrous tumor. An update on clinical, molecular and diagnostic features. Ann Diagn Pathol. 2018; 34:142-150. https://doi.org/10.1016/j.anndiagpath.2018.01.0 $\underline{04}$

22. Kao YC, Lin PC, Yen SL, et al. Clinicopathological and genetic heterogeneity of the head and neck solitary fibrous tumours: a comparative histological, immunohistochemical and molecular study of 36 cases. Histopathology. 2016; 68(4):492-501. https://doi.org/10.1111/his.12772

23. Kant $\mathrm{R}$, Davis A, Verma V. Thyroid nodules: advances in evaluation and management. $\mathrm{Am}$ Fam Physician. 2020; 102(5):298-304. PMID:32866364

24. Ning S, Song $X$, Xiang $L$, Chen $Y$, Cheng $Y$, Chen $\mathrm{H}$. Malignant solitary fibrous tumor of the thyroid gland: report of a case and review of 
the literature. Diagn Cytopathol. 2011; 39(9):694-699.

https://doi.org/10.1002/dc.21538

25. Parwani AV, Galindo R, Steinberg DM, Zeiger MA, Westra WH, Ali SZ. Solitary fibrous tumor of the thyroid: cytopathologic findings and differential diagnosis. Diagn Cytopathol. 2003; 28(4):213-216. https://doi.org/10.1002/dc. 10264

26. Tani $\mathrm{E}$, Wejde J, Åström $\mathrm{K}$, Wingmo IL, Larsson O, Haglund F. FNA cytology of solitary fibrous tumors and the diagnostic value of STAT6 immunocytochemistry. Cancer Cytopathol. 2018; 126(1):36-43. https://doi.org/10.1002/cncy.21923

27. Tanahashi J, Kashima K, Daa T, et al. Solitary fibrous tumor of the thyroid gland: report of two cases and review of the literature. Pathol Int. 2006; 56(8):471-477. https://doi.org/10.1111/j.14401827.2006.01992.x

28. Cameselle-Teijeiro J, Manuel Lopes J, Villanueva JP, Gil-Gil P, Sobrinho-Simões M. Lipomatous haemangiopericytoma (adipocytic variant of solitary fibrous tumour) of the thyroid. Histopathology. 2003; 43(4):406-408. https://doi.org/10.1046/i.13652559.2003.01696.x

29. Koelsche $C$, Schweizer L, Renner M, et al. Nuclear relocation of STAT6 reliably predicts NAB2-STAT6 fusion for the diagnosis of solitary fibrous tumour. Histopathology. 2014; 65(5):613-622.

https://doi.org/10.1111/his.12431

30. Cameselle-Teijeiro J. Uncommon tumors of the thyroid gland. Int J Surg Pathol. 2010; 18(3 Suppl):S205-208.

https://doi.org/10.1177/1066896910370885

31. Corrado S, Corsello SM, Maiorana A, et al. Papillary thyroid carcinoma with predominant spindle cell component: report of two rare cases and discussion on the differential diagnosis with other spindled thyroid neoplasm. Endocr Pathol. 2014; 25(3):307-314. https://doi.org/10.1007/s12022-013-9290-7

32. Takada N, Hirokawa M, Ito $M$, et al. Papillary thyroid carcinoma with desmoid-type fibromatosis: A clinical, pathological, and immunohistochemical study of 14 cases. Endocr J. 2017; 64(10):1017-1023. https://doi.org/10.1507/endocri.EJ17-0242

33. Folpe AL, Lloyd RV, Bacchi CE, Rosai J. Spindle epithelial tumor with thymus-like differentiation: a morphologic, immunohistochemical, and molecular genetic study of 11 cases. Am J Surg Pathol. 2009; 33(8):1179-1186.
https://doi.org/10.1097/PAS.0b013e31819e61c 8

34. Chen G, Liu Z, Su C, et al. Primary peripheral nerve sheath tumors of the thyroid gland: $A$ case report and literature review. Mol Clin Oncol. 2016; 4(2):209-210. https://doi.org/10.3892/mco.2015.703

35. Zhang $\mathrm{Y}$, Tang $\mathrm{H}$, $\mathrm{Hu} \mathrm{H}$, Yong $X$. A rare primary tumor of the thyroid gland: a new case of leiomyoma and literature review. Clin Med Insights Oncol. 2018; 12:1179554918813535. https://doi.org/10.1177/1179554918813535

36. Canu GL, Bulla JS, Lai ML, et al. Primary thyroid leiomyosarcoma: a case report and review of the literature. G Chir. 2018; 39(1):5156.

https://doi.org/10.11138/gchir/2018.39.1.051

37. Miettinen M. Smooth muscle tumors of soft tissue and non-uterine viscera: biology and prognosis. Mod Pathol. 2014; 27(1 Suppl):S1729. https://doi.org/10.1038/modpathol.2013.178

38. Kandil E, Abdel Khalek M, Abdullah O, et al. Primary peripheral nerve sheath tumors of the thyroid gland. Thyroid. 2010; 20(6):583-586. https://doi.org/10.1089/thy.2009.0245

39. Dhar H, Dabholkar JP, Kandalkar BM, Ghodke R. Primary thyroid schwannoma masquerading as a thyroid nodule. J Surg Case Rep. 2014; 9:rju094. https://doi.org/10.1093/jscr/rju094

40. Miettinen M, McCue PA, Sarlomo-Rikala $M$, et al. Sox10--a marker for not only schwannian and melanocytic neoplasms but also myoepithelial cell tumors of soft tissue: a systematic analysis of 5134 tumors. Am J Surg Pathol. 2015; 39(6):826-835. https://doi.org/10.1097/PAS.000000000000039 $\underline{8}$

41. Bohlok A, El Khoury M, Bormans A, et al. Schwannoma of the colon and rectum: a systematic literature review. World J Surg Oncol. 2018; 16(1):125-136. https://doi.org/10.1186/s12957-018-1427-1

42. Ghasemi-Rad $M$, Wang $K Y$, Jain $S$, Lincoln CM. Solitary fibrous tumor of thyroid: a case report with review of literature. Clin Imaging. 2019; 53:105-107. https://doi.org/10.1016/j.clinimag.2018.09.011

43. Baldi GG, Stacchiotti S, Mauro V, et al. Solitary fibrous tumor of all sites: outcome of late recurrences in 14 patients. Clin Sarcoma Res. 2013; 3:4. https://doi.org/10.1186/2045-3329-34

44. Colia V, Provenzano S, Morosi C, et al. Solitary fibrous tumour presenting with a single bone metastasis: report of six cases and literature review. Clin Sarcoma Res. 2016; 6(1):16. https://doi.org/10.1186/s13569-016-0055-1 
45. Demicco EG, Park MS, Araujo DM, et al. Solitary fibrous tumor: a clinicopathological study of 110 cases and proposed risk assessment model. Mod Pathol. 2012; 25(9):1298-1306.

https://doi.org/10.1038/modpathol.2012.83
46. Salas S, Resseguier N, Blay JY, et al. Prediction of local and metastatic recurrence in solitary fibrous tumor: construction of a risk calculator in a multicenter cohort from the French Sarcoma Group (FSG) database. Ann Oncol. 2017; 28(8):1979-1987. https://doi.org/10.1093/annonc/mdx250 